Journal of Engineering and Science Research 2 (2): 52-58, 2018

e-ISSN: 2289-7127

(C) RMP Publications, 2018

DOI: $10.26666 / \mathrm{rmp}$.jesr.2018.2.8

\title{
Design of Gear-train based Sliding-mode Triboelectric Nanogenerator and its Hybridization Perspective with Perovskite Solar Cell
}

Jae Hyun Kim ${ }^{a}$, Wook Kim ${ }^{b}$, Sera Kwon ${ }^{c}$, Kwun-Bum Chung ${ }^{c}$, Hojin Lee ${ }^{d}$, and Dukhyun Choi ${ }^{b}$

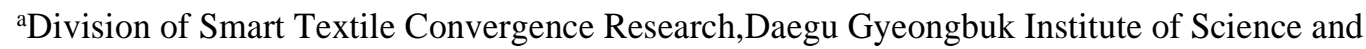
Technology (DGIST), 333, Techno Jungang Daero, Hyeonpung, Daegu, 42988, Republic of Korea

${ }^{b}$ Department of Mechanical Engineering, College of Engineering, Kyung Hee University,

1732 Deogyeong-daero, Giheung-gu, Yongin-si, Gyeonggi-do, 17104, Republic of Korea ${ }^{c}$ Division of Physics and Semiconductor Science, Dongguk University, Seoul, 100-715, Korea

${ }^{\mathrm{d} S}$ School of Electronic Engineering, Soongsil University, Seoul, 156-743, Korea

\begin{abstract}
Triboelectric nanogenerator (TENG) is a renewable energy generation technology that can convert mechanical energy of various forms like wind, tides, or vibrations into usable electrical energy. The output performance of the TENG depends on several parameters such as the amount of input energy, material selection, environmental conditions, etc. If the amount of input energy is low, the output power is reduced. Furthermore, if the input frequency $\left(f_{\text {in }}\right)$ is low, not only is the output power reduced, but also the working frequency $\left(f_{w}\right)$ of the TENG is lowered. This cumulative undesirable effect lowers the output energy from the TENG within a specific time period. Therefore, in this work we employed a gear mechanism with the TENG in order to increase its working frequency, and systematically studied its effects on the TENG output. The gear mechanism consists of the inputgear, which receives the input mechanical energy, and the working-gear, which transfers the input energy to the TENG. By reducing the size of the working-gear, it was possible to increase the TENG output frequency. We fixed the input frequency at $1.5,3$, and $4.5 \mathrm{~Hz}$ and controlled the gear-ratio as 1 and 1.7. Under input frequency of $4.5 \mathrm{~Hz}$, we could enhance the TENG output current density by 1.6 times using a gear-ratio of 1.7 . Thus, by means of this kinematic system, we were able to increase the working frequency and current output of the TENG. We expect that our gear-train based TENG will help provide high output even under low frequency input energy conditions, and that it will be effectively utilized in practical TENG systems. We also expect that the hybridization of perovskite solar cell and the TENG is a promising energy scavenger.
\end{abstract}

Key words: Triboelectric nanogenerator, Gear-train, Hybridization, Perovskite solar cell

\section{INTRODUCTION}

Triboelectric nanogenerators (TENG) are devices that covert ambient mechanical energy in various forms like human motion, wind, tides, or vibrations into usable electrical energy [1-3]. They can effectively convert even low-level input energy into electricity and can be applied to all forms of mechanical inputs. Thus, they have the potential to reliably power wearable devices, medical devices, sensors, etc [4-6]. The TENG operation is based on static electrification and charge induction [7-10]. Static electrification is a phenomenon where two materials come into contact and exchange surface charges. This then results in one material carrying a positive surface charge and the other material a negative surface charge. The type of charge that develops on the material surface depends on differences in the surface energy of the materials coming into contact [11-12]. A potential difference develops between the two material surfaces due to charge separation. The resulting electric field can then be utilized to convert any material motion into electron flow between electrodes attached to the surface charged materials. The electrical energy output depends on several parameters such as temperature, humidity, contact area, surface pattern, motion speed, frequency, etc. Thus, the electrical output from the TENG can be enhanced by careful control of these

Corresponding Author: Dukhyun Choi, Department of Mechanical Engineering, Kyung Hee University, 1732 Deogyeong-daero, Giheung-gu, Yongin-si, Gyeonggi-do, 17104, Republic of Korea, +82-31-201-3320 
parameters [13-17]. Some previous studies have reported high TENG outputs, however they used high input energy levels such as high speed input rotation of $1000 \mathrm{rpm}$, sliding speeds of $10 \mathrm{~m} / \mathrm{s}$, and high input forces of $600 \mathrm{~N}$ [18-19]. Ambient mechanical energy, on the contrary, is irregular and does not maintain such high input levels. Thus, when operating a TENG in practical conditions, due to low input forces and frequencies, the TENG output is also low. In order to exploit the input energy more effectively, we can use gear based kinematic mechanisms for conversions in torque and angular velocity. If the input-gear is larger than the working-gear, the angular velocity of the working-gear will be greater according to the gearratio. Based on this concept, in this work we studied a gear-train based TENG system. We experimentally investigated the effects of changing the gear-ratio on the TENG output for fixed input frequency. The diving-gear received the input energy and rotated with the same frequency as the input frequency. The working-gear, due its smaller radius, rotated with a higher frequency than the input-gear, and finally transferred this energy to the TENG. The TENG, following the working-gear, can thus move with an increased speed, leading to higher electric output. In this work, we used gear-ratio of 1 and 1.7 for comparing the TENG output. We confirmed that TENG output increased on increasing its working frequency. Our work can provide a design direction for improved TENG based energy harvesting systems.

\section{EXPERIMENTAL}

\section{TENG device fabrication}

The TENG device was fabricated before assembling the TENG system. Aluminum (Al) film of $200 \mu \mathrm{m}$ thickness was used as the positively charged material and as the electrode material. Polyimide film of $200 \mu \mathrm{m}$ thickness was used as the negatively charged material. For bottom layer electrode, the Al film was attached below the polyimide film by means of an adhesive. The size of the fabricated device was $4 \mathrm{~cm} \times 1 \mathrm{~cm}$, and the TENG was driven in lateral contact-sliding mode.

\section{TENG kinematic system assembly}

The fabricated TENG device and gear-train was connected by means of a slider-crank mechanism. The input-gear was first connected to a DC motor, then the working-gear of smaller size was connected to the inputgear. A crank linked the axis of the working-gear to a connecting rod with a slider at its end. In this way gear rotation motion was converted into translational motion. Below the slider, the top electrode $\mathrm{Al}$ film of the TENG device was attached. On top of the guide, upon which the slider moved, two polyimide films with bottom $\mathrm{Al}$ electrode were attached about $6 \mathrm{~mm}$ apart. Thus, the total travel distance of the slider on the guide was 26 $\mathrm{mm}$. The slider and guide were fabricated using 3D printing. A gap of $0.3 \mathrm{~mm}$ was maintained between the slider and guide in order to avoid unwanted interference.

\section{Measurement and analysis}

Two gear-trains with gear-ratio 1 and 1.7 were used for TENG output comparison. The input frequency was controlled at $1.5,3$, and $4.5 \mathrm{~Hz}$ to study its effects on the working frequency and TENG output. A DC motor (GM35D-3657) was used to provide input at the inputgear. A DC power supply (APS3005S, Atten) was used to supply power to the DC motor. The TENG output was measured using an oscilloscope (MDO3052, Tektronix) with $40 \mathrm{M} \Omega$ input resistance probe (P5100A, Tektronix). In order to measure the TENG output current, a lownoise current preamplifier (SR570, Stanford Research Systems) was connected to the oscilloscope.

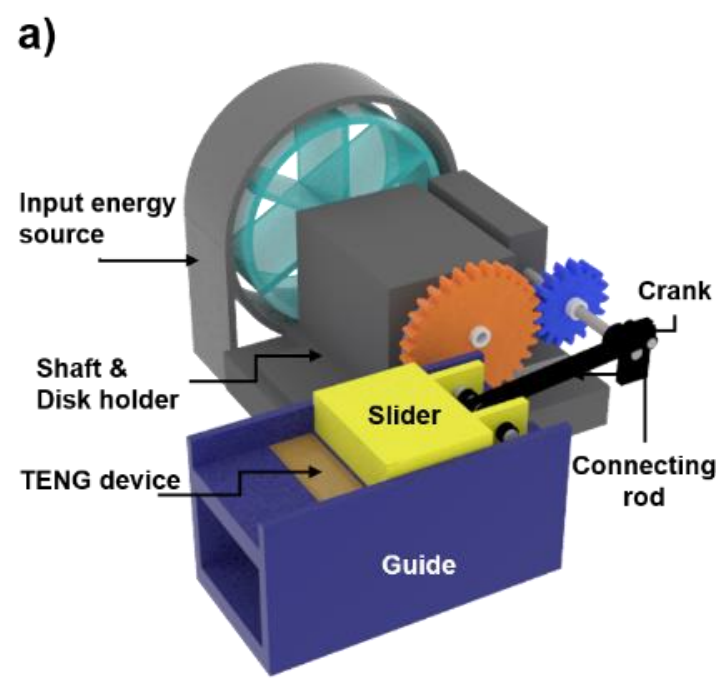




\section{b) Gear train}
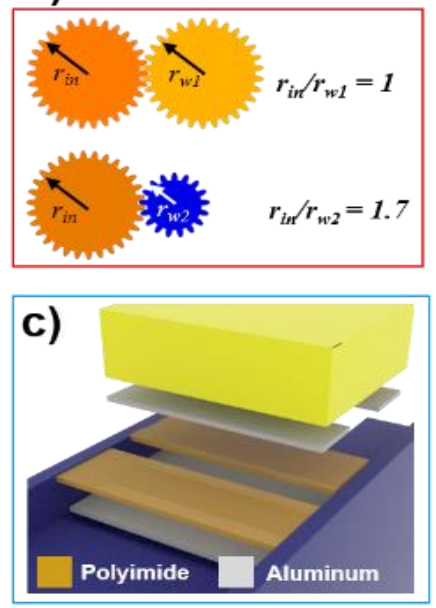

Fig 1 Components of the TENG kinematic system.

(a) Schematic diagram of the gear-train and slider-crank mechanism based TENG system. (b) Utilized gear-trains and their corresponding gear-ratios. (c) TENG device structure.

\section{RESULTS AND DISCUSSION}

Figure 1 shows TENG kinematic system design schematic with the TENG device installed. As shown in figure $1 \mathrm{a}$, the system design can be divided into two parts. In the first part, the kinematic system transfers the input energy onto the TENG device in real-time while also increasing its working frequency. In the second part, the TENG device that is arranged on the guide, converts the mechanical sliding motion into electrical energy. The generated electrical output from the TENG device can be finally be transferred to an external circuit. As shown in figure $1 \mathrm{~b}$, two gear-trains with gear-ratios 1 and 1.7 were used in the kinematic system. The inputgear which receives the input energy has radius $r_{i n}$ and the working-gear has radius $r_{w}$. If $r_{i n}>r_{w}$, then the gearratio, $r_{i n} / r_{w}>1$, and correspondingly the working frequency, $f_{w}$, is then greater than the input frequency, $f_{\text {in. }}$. As shown in figure $1 c$, the TENG device operates in lateral contact-sliding mode, by utilizing the different charging characteristics of polyimide and aluminum films. The aluminum film attached on the slider serves as the top electrode and positively charged material. Polyimide film attached on the guide serves as the negatively charged material with an aluminum film attached to its bottom serving as the bottom electrode.

Figure 2a shows photograph of the final TENG kinematic system. The slider and guide were fabricated using 3D printing. A DC motor was used to provide input rotation force to the system. In order to ensure smooth movement of the slider, the height of the guide was set equal to the center of rotation of the gear-train. In order to avoid contact between aluminum film on the slider and the top of guide, a gap of $0.3 \mathrm{~mm}$ was maintained between the slider and the guide. Through slider-crank mechanism analysis, the displacement of the slider can be given as,

$$
x \approx r\left[(1-\cos \theta)+\frac{1}{2}\left(\frac{r}{l}\right) \sin ^{2} \theta\right]
$$

where $x$ is the displacement of the slider, $\theta$ is the angle between the crank and slider motion axis, $r$ is the length of crank, and $l$ is the length of connecting-rod. Under minimum slider displacement, $\theta=0^{\circ}$, thus $x=0$. Under maximum slider displacement, $\theta=180^{\circ}$, thus

$$
x \approx 2 r
$$

Therefore, the maximum slider displacement is defined as twice the crank length. Since the crank we used had length of $13 \mathrm{~mm}$, the maximum displacement of the slider was confirmed to be $26 \mathrm{~mm}$ based on the above design condition. Accordingly, the TENG device width was set as $10 \mathrm{~mm}$ each and the distance between the devices was set as $6 \mathrm{~mm}$ as shown in Figure 2b.

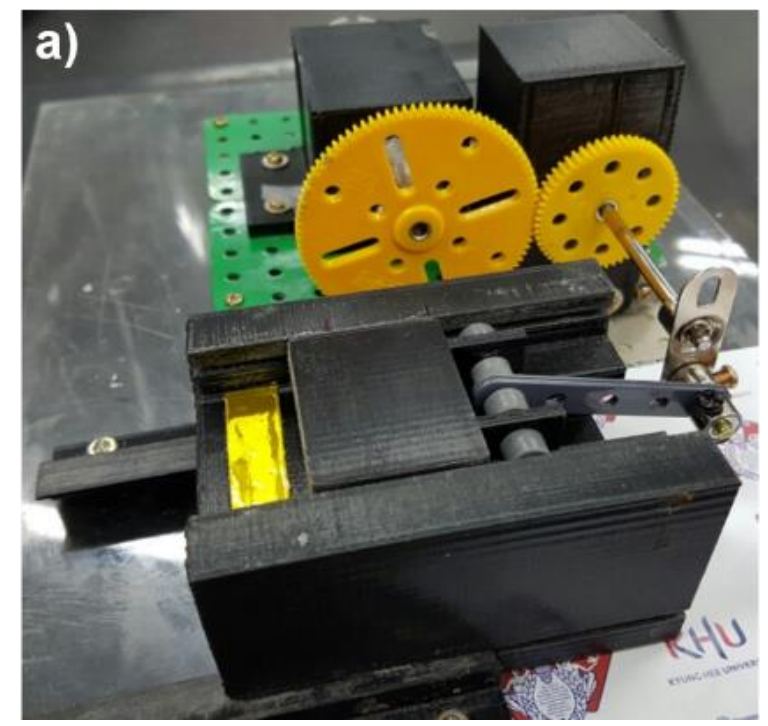




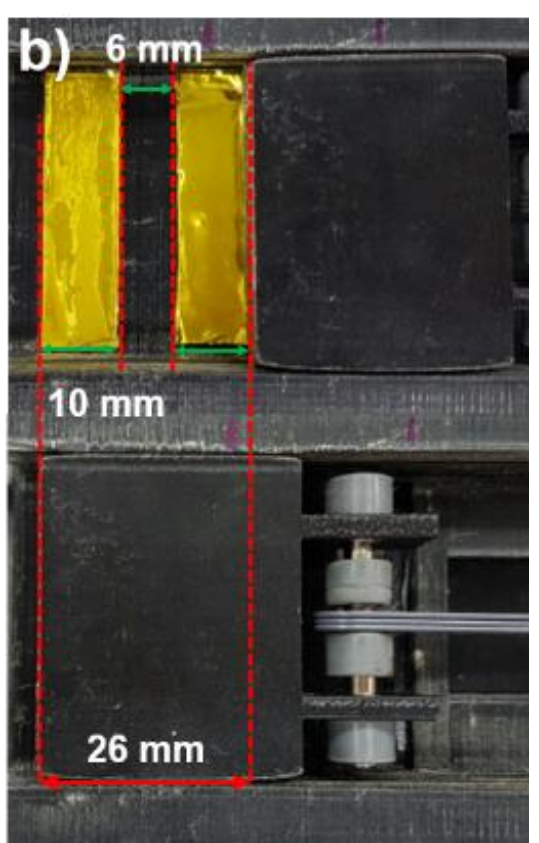

Fig 2 Final TENG kinematic system. (a) Photograph of the actual gear-train and slider-crank mechanism based TENG system. (b) TENG device dimensions and maximum travel distance of the slider on the guide.

Figure 3 shows the fundamental mechanism of lateral sliding-contact mode TENG device. In the initial state shown in figure $3 \mathrm{a}$, the top $\mathrm{Al}$ film and polyimide film are in complete contact. Due to differences in the surface energy levels of the two materials, electrons transfer from Al to polyimide and persist on its surface. As shown in figure $3 \mathrm{~b}$, when the top $\mathrm{Al}$ film slides to the right, electrons move from the bottom $\mathrm{Al}$ film to the top Al film in order to maintain charge neutrality. When the top Al film and polyimide film are fully separated as in figure $3 \mathrm{c}$, all the positive charge has moved from top $\mathrm{Al}$ film to the bottom $\mathrm{Al}$ film. As the top $\mathrm{Al}$ film slides left electrons return from the top Al film to bottom Al film, as shown in figure $3 \mathrm{~d}$. Thus, the TENG device converts sliding motion into alternating current electrical output.

Figure 4 shows that the TENG device electrical current output depends on the input frequency. In order to ensure that the gear does not influence the output, the gear-ratio, $r_{i n} / r_{w}$ was set equal to 1 , and TENG current output was measured at input frequency $1.5 \mathrm{~Hz}, 3.0 \mathrm{~Hz}$ and $4.5 \mathrm{~Hz}$. The measured output current density increased with increase in frequency as $0.1 \mu \mathrm{A} / \mathrm{cm}^{2}$ at $1.5 \mathrm{~Hz}, 0.25 \mu \mathrm{A} / \mathrm{cm}^{2}$ at $3 \mathrm{~Hz}$ and $0.35 \mu \mathrm{A} / \mathrm{cm}^{2}$ at $4.5 \mathrm{~Hz}$. This trend of increase in TENG current output with increase in input frequency is consistent with results reported in previous research [20-21]. This is because increase in input frequency corresponds to increase in speed of sliding motion and electron flow. Based on this reasoning we recognize that a gear mechanism is well suited for use with the TENG to improve its output.

Before measuring the effects of increasing gear-ratio on the TENG output, we first develop a theoretical understanding for how working frequency can be changed using the gear-ratio. The relationship between working frequency $\left(f_{w}\right)$, input frequency $\left(f_{i n}\right)$ and gear-ratio is given as,

$$
f_{w}=\frac{r_{\text {in }}}{r_{w}} f_{\text {in }}
$$

For gear-ratio of 1 , the working frequency will be same as the input frequency. Thus we can see in figure 4 that the TENG output frequency or the working frequency is the same as the input frequency (i.e. $\mathrm{f}_{\mathrm{w}}=1.5 \mathrm{~Hz}, 3 \mathrm{~Hz}, 4.5 \mathrm{~Hz}$ ). If the gear-ratio is increased to 1.7 , the working frequencies will become $2.55 \mathrm{~Hz}$ ( $\left.f_{\text {in }}=1.5 \mathrm{~Hz}\right), 5.1 \mathrm{~Hz}\left(\mathrm{f}_{\mathrm{in}}=3 \mathrm{~Hz}\right)$, and $7.65 \mathrm{~Hz}\left(\mathrm{f}_{\mathrm{in}}=4.5 \mathrm{~Hz}\right)$. The actual measured working frequencies were $2.5 \mathrm{~Hz}$, $4.8 \mathrm{~Hz}$, and $7.2 \mathrm{~Hz}$ which are about $6 \%$ lower than the theoretical values. This can be attributed to damping losses due to frictional sliding and gear-coupling.

Figure 5 shows TENG current density output for gear-ratios 1 and 1.7 under input frequencies $1.5 \mathrm{~Hz}$, $3 \mathrm{~Hz}$ and $4.5 \mathrm{~Hz}$. In general, the results confirm that working frequency greater than the input frequency can be obtained for gear-ratio of 1.7. The TENG output results are summarized in figure $5 \mathrm{~d}$ for comparison. We can see that on average 1.5 times current density enhancement could be obtained by increasing the gearratio form 1 to 1.7. The highest TENG current density obtained was $0.6 \mu \mathrm{A} / \mathrm{cm}^{2}$ at $\mathrm{f}_{\text {in }}=4.5 \mathrm{~Hz}$. Even under high input frequency of $4.5 \mathrm{~Hz}$, we could obtain enhanced and stable TENG output. Thus, we conclude that the TENG output level as well as its output frequency can be reliably increased by appropriate control of gearratio.

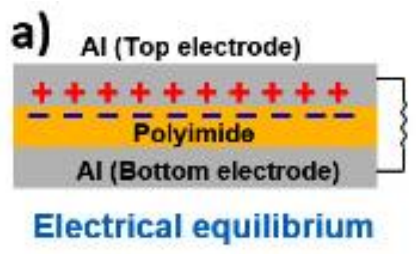



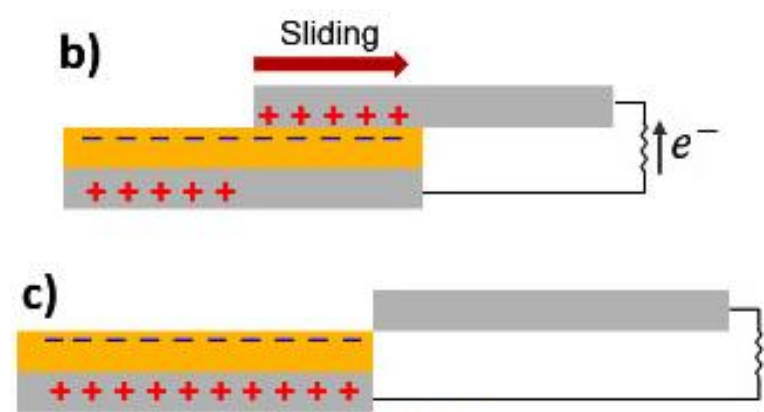

Electrical equilibrium

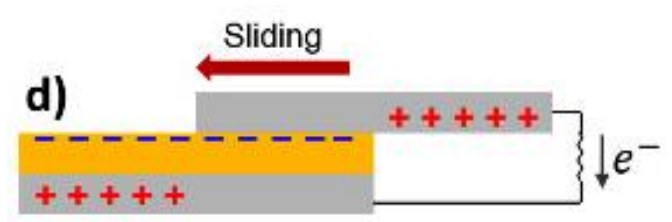

Fig 3 Fundamental mechanism of lateral sliding-contact mode TENG device. (a) Initial state of TENG device.

(b) Right sliding of top $\mathrm{Al}$ film and electron motion between electrodes. (c) Fully separated TENG device. (d) Left sliding of top $\mathrm{Al}$ film and electron motion between electrodes.

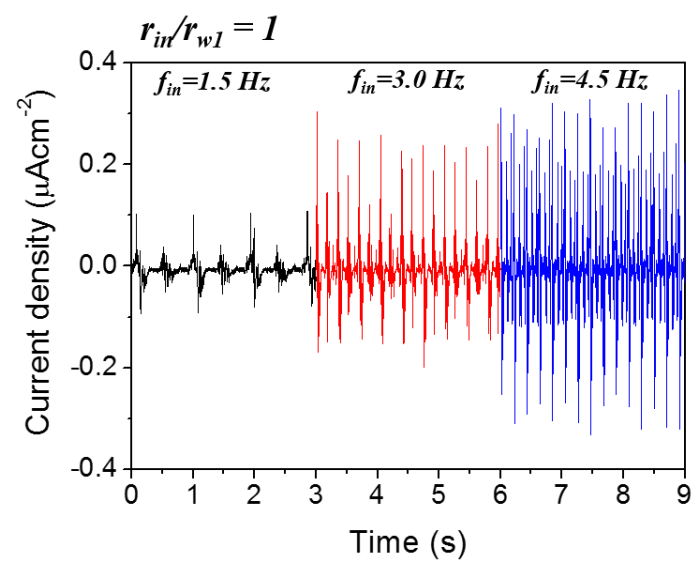

Fig 4 TENG output dependence on input frequency.

Figure 6 shows TENG current density output for input frequency $4.5 \mathrm{~Hz}$ and gear-ratio 1 was similar to output for input frequency $3 \mathrm{~Hz}$ and gear-ratio 1.7. This was because the final working frequency in both cases was similar, that is, $4.5 \mathrm{~Hz}$ and $4.8 \mathrm{~Hz}$ respectively. This shows that by appropriate design of gear-ratio, we can help maintain stable TENG output level even when the input frequency decreases. The relationship between TENG output and working frequency can also be used for predictive control purposes.

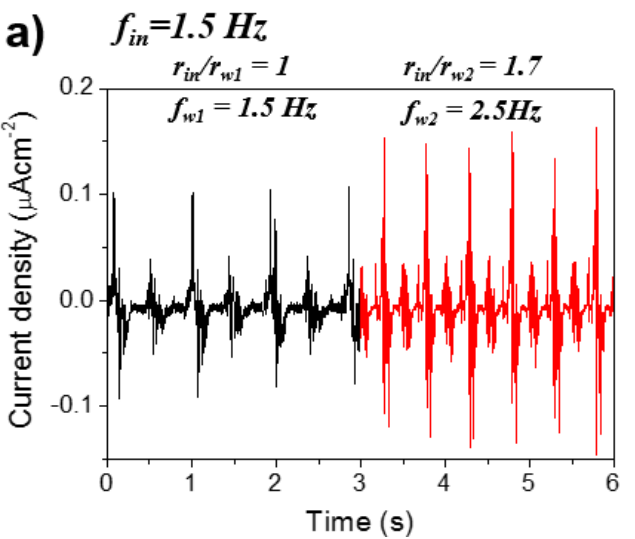

b) $f_{\text {in }}=3.0 \mathrm{~Hz}$

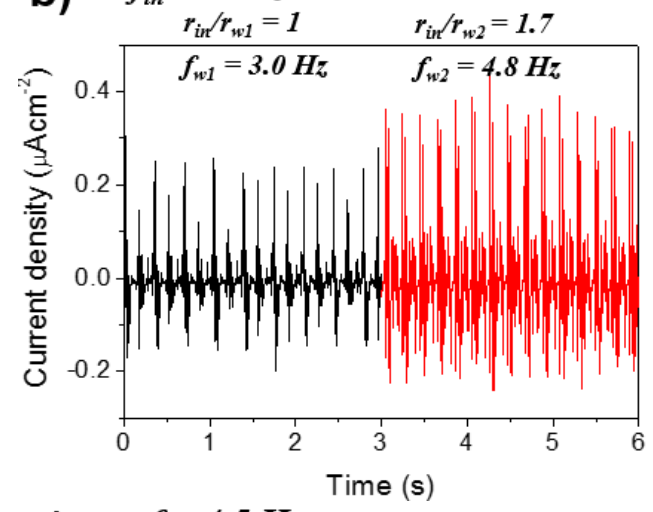

c) $f_{\text {in }}=4.5 \mathrm{~Hz}$
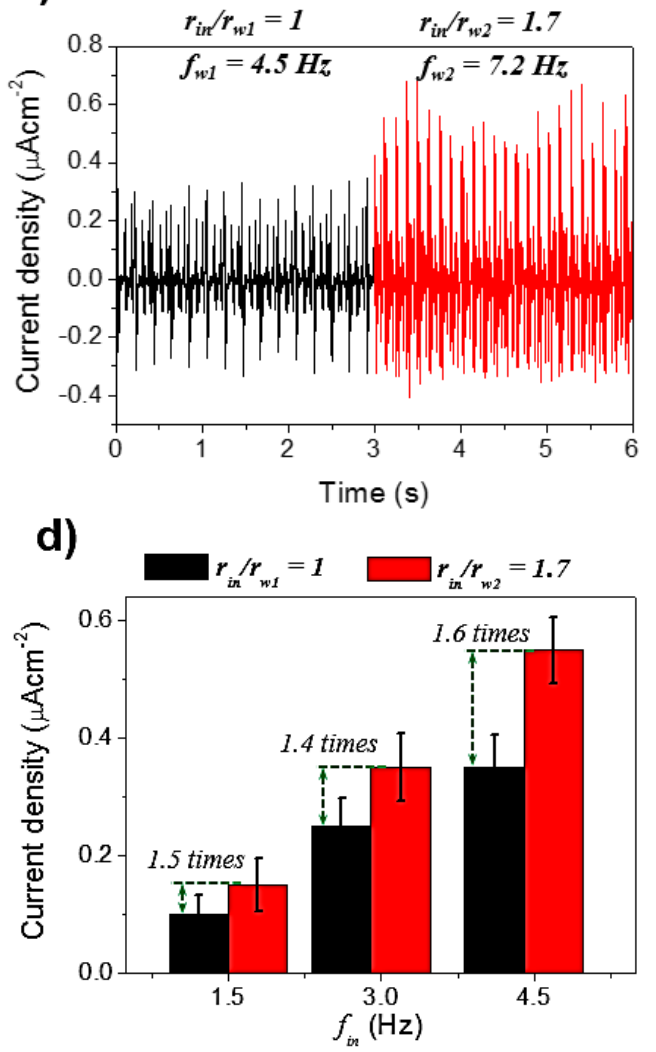
Fig 5 Measured TENG current for gear-ratios 1 and 1.7 under input frequency of (a) $1.5 \mathrm{~Hz}$, (b) $3 \mathrm{~Hz}$, and (c) $4.5 \mathrm{~Hz}$.

(d) Comparing current enhancement due to higher gear-ratio.

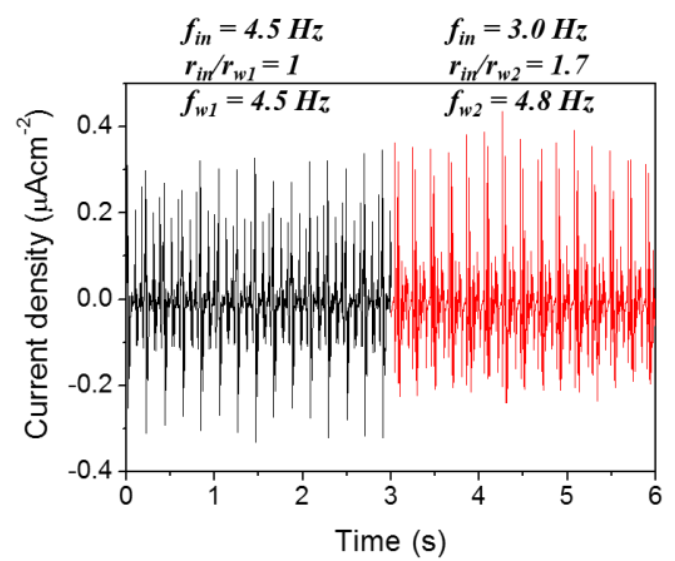

Fig 6 Similar TENG output working frequency obtained using different input frequencies and gear-ratios.

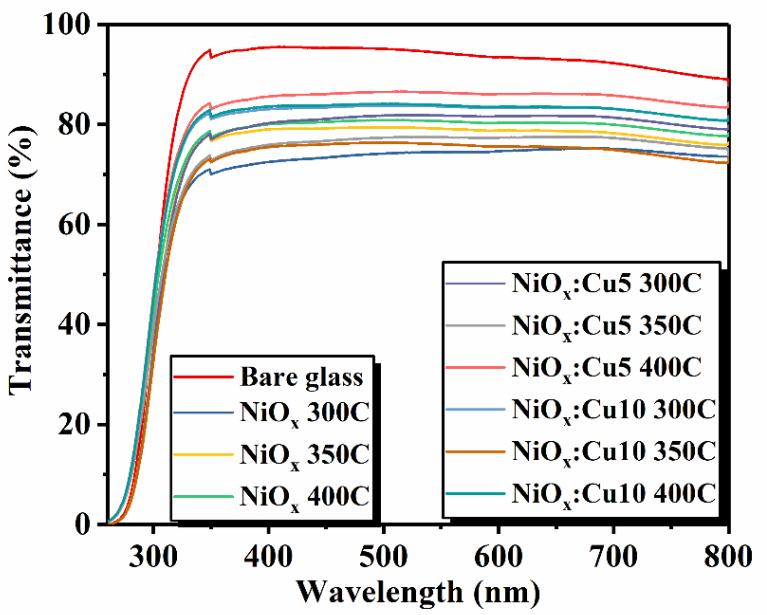

Fig 7 Transmittance spectra of undoped and $\mathrm{Cu}$ doped $\mathrm{NiO}_{\mathrm{x}}$ nanoparticles: $\mathrm{NiO}_{\mathrm{x}} 300 \mathrm{C}, \mathrm{NiO}_{\mathrm{x}} 350 \mathrm{C}$ and $\mathrm{NiO}_{\mathrm{x}} 400 \mathrm{C}$ mean that $\mathrm{NiO}_{\mathrm{x}}$ is calcined at $300{ }^{\circ} \mathrm{C}, 350^{\circ} \mathrm{C}$, and $400{ }^{\circ} \mathrm{C}$. $\mathrm{NiO}_{\mathrm{x}}: \mathrm{Cu} 5$ $300 \mathrm{C}, \mathrm{NiO}_{\mathrm{x}}: \mathrm{Cu} 5350 \mathrm{C}$ and $\mathrm{NiO}_{\mathrm{x}}$ : $\mathrm{Cu} 5400 \mathrm{C}$ do that $5 \% \mathrm{Cu}-$ doped $\mathrm{NiO}_{\mathrm{x}}$ is at $300^{\circ} \mathrm{C}, 350^{\circ} \mathrm{C}$, and $400^{\circ} \mathrm{C}$. $\mathrm{NiO}_{\mathrm{x}}: \mathrm{Cu} 10300 \mathrm{C}$, $\mathrm{NiO}_{x}: \mathrm{Cu} 10350 \mathrm{C}$ and $\mathrm{NiO}_{\mathrm{x}}$ : $\mathrm{Cu} 10400 \mathrm{C}$ do that $10 \% \mathrm{Cu}$-doped $\mathrm{NiO}_{\mathrm{x}}$ is at $300^{\circ} \mathrm{C}, 350^{\circ} \mathrm{C}$, and $400^{\circ} \mathrm{C}$.

The TENG system is excellent for scavenging alternative energy in the environment that the solar energy is insufficient to provide enough energy. The concept of a hybridized energy harvester incorporating solar cell and TENG is promising way to use energy sources effectively and complementarily. The perovskite solar cell is one of the most prominent among high efficient solar cells. For enhancing the efficiency of perovskite solar cell, the hole transporting layer is very important to extract generated holes to electrode. $\mathrm{Cu}-$ doped $\mathrm{NiO}_{\mathrm{x}}$ layer is an excellent hole transporting layer.
One of main criteria of the layer is transmittance. With varying composition of $\mathrm{Cu}$ in $\mathrm{NiO}_{\mathrm{x}}$, the maximum transmittance is obtained in $5 \% \mathrm{Cu}$-doped $\mathrm{NiO}_{\mathrm{x}}$ in which the calcination temperature is $400{ }^{\circ} \mathrm{C}$ as shown in Fig. 7. This good transmittance will provide a good cell performance. The hybridization of the perovskite solar cell and the TENG system will open a very perspective energy source world. The detailed results and discussion is under studying and will be reported later.

\section{CONCLUSION}

In this work a gear-train and slider-crank mechanism based TENG system was designed, and the TENG output working frequency was enhanced through appropriate control of the input-gear to working-gear gear-ratio. We carefully designed the kinematic system suitable for a lateral sliding-contact mode TENG device. By increasing the input frequency to the TENG we could both increase the output frequency as well as increase the output level. We believe that our proposed system design can increase TENG output under low frequency input conditions. Thus, we expect our results will help provide a design direction for practical TENG based energy harvesting systems. We also suggested that the hybridization of perovskite solar cell and the TENG is an excellent strategy to collecting multiple types of energies from the environment.

\section{ACKNOWLEDGMENTS}

This research was supported by Global Infrastructure Program through the National Research Foundation of Korea(NRF) funded by the Ministry of Science and ICT(NRF-2016K1A3A1A09917863).

\section{REFERENCES}

[1] Fan, X., Chen, J., Yang, J., Bai, P., Li, Z., and Wang, Z. L. 2015. Ultrathin, rollable, paper-based triboelectric nanogenerator for acoustic energy harvesting and self-powered sound recording. ACS Nano, 9, 4236-4243.

[2] Chen, J., Yang, J., Li, Z., Fan, X., Zi, Y., Jing, Q., Guo, H., Wen, Z., Pradel, K. C., Niu, S., and Wang, Z. L. 2015. Networks of triboelectric nanogenerators for harvesting water wave energy: a potential approach toward blue energy. ACS Nano, 9, 33243331. 
[3] Yang, W., Chen, J., Zhu, G., Yang, J., Bai, P., Su, Y., Jing, Q., Cao, X., and Wang, Z. L. 2013. Harvesting energy from the natural vibration of human walking. ACS Nano, 7, 11317-11324.

[4] Zheng, Q., Shi, B., Fan, F., Wang, X., Yan, L., Yuan, W., Wang, S., Liu, H., Li, Z., and Wang, Z. L. 2014. In vivo powering of pacemaker by breathing- driven implanted triboelectric nanogenerator. Advanced Materials, 26, 5851-5856.

[5] Zhang, X. S., Han, M. D., Wang, R. X., Zhu, F. Y., Li, Z. H., Wang, W., and Zhang, H. X. 2013. Frequency-multiplication high-output triboelectric nanogenerator for sustainably powering biomedical microsystems. Nano letters, 13, 1168-1172.

[6] Han, C. B., Zhang, C., Li, X. H., Zhang, L., Zhou, T., Hu, W., and Wang, Z. L. 2014. Self-powered velocity and trajectory tracking sensor array made of planar triboelectric nanogenerator pixels. Nano Energy, 9, 325-333.

[7] Wang, Z. L. 2015. Triboelectric nanogenerators as new energy technology and self-powered sensorsPrinciples, problems and perspectives. Faraday discussions, 176, 447-458.

[8] Wang, Z. L. 2013. Triboelectric nanogenerators as new energy technology for self-powered systems and as active mechanical and chemical sensors. ACS Nano, 7, 9533-9557.

[9] Zhu, G., Peng, B., Chen, J., Jing, Q., and Wang, Z. L. 2015. Triboelectric nanogenerators as a new energy technology: From fundamentals, devices, to applications. Nano Energy, 14, 126-138.

[10] Wang, Z. L., Chen, J., and Lin, L. 2015. Progress in triboelectric nanogenerators as a new energy technology and self-powered sensors. Energy and Environmental Science, 8, 2250-2282.

[11] Lacks, D. J., and Sankaran, R. M. 2011. Contact electrification of insulating materials. Journal of Physics D: Applied Physics, 44, 453001.

[12] Zhou, Y. S., Wang, S., Yang, Y., Zhu, G., Niu, S., Lin, Z. H., Liu, Y., and Wang, Z. L. 2014. Manipulating nanoscale contact electrification by an applied electric field. Nano letters, 14, 1567-1572.
[13] Nguyen, V., and Yang, R. 2013. Effect of humidity and pressure on the triboelectric nanogenerator. Nano Energy, 2, 604-608.

[14] Fan, F. R., Lin, L., Zhu, G., Wu, W., Zhang, R., and Wang, Z. L. 2012. Transparent triboelectric nanogenerators and self-powered pressure sensors based on micropatterned plastic films. Nano letters, 12, 3109-3114.

[15] Lee, K. Y., Yoon, H. J., Jiang, T., Wen, X., Seung, W., Kim, S. W., and Wang, Z. L. 2016. Fully Packaged Self- Powered Triboelectric Pressure Sensor Using Hemispheres- Array. Advanced Energy Materials, 6.

[16] Wen, X., Su, Y., Yang, Y., Zhang, H., and Wang, Z. L. 2014. Applicability of triboelectric generator over a wide range of temperature. Nano Energy, 4, 150-156.

[17] Wang, S., Lin, L., and Wang, Z. L. 2012. Nanoscale triboelectric-effect-enabled energy conversion for sustainably powering portable electronics. Nano letters, 12, 6339-6346.

[18] Zhu, G., Lin, Z. H., Jing, Q., Bai, P., Pan, C., Yang, Y., Zhou, Y., and Wang, Z. L. 2013. Toward largescale energy harvesting by a nanoparticle-enhanced triboelectric nanogenerator. Nano letters, 13, 847853.

[19] Zhu, G., Zhou, Y. S., Bai, P., Meng, X. S., Jing, Q., Chen, J., and Wang, Z. L. 2014. A Shape- Adaptive Thin- Film- Based Approach for 50\% HighEfficiency Energy Generation Through MicroGrating Sliding Electrification. Advanced Materials, 26, 3788-3796.

[20] Wang, S., Lin, L., Xie, Y., Jing, Q., Niu, S., and Wang, Z. L. 2013. Sliding-triboelectric nanogenerators based on in-plane charge-separation mechanism. Nano letters, 13, 2226-2233.

[21] Niu, S., Liu, Y., Wang, S., Lin, L., Zhou, Y. S., Hu, Y., and Wang, Z. L. 2013. Theory of sliding- mode triboelectric nanogenerators. Advanced materials, 25, 6184-6193. 